\title{
MONITORING AQUATIC WEEDS IN INDIAN WETLANDS USING MULTITEMPORAL REMOTE SENSING DATA WITH MACHINE LEARNING TECHNIQUES
}

\author{
Vahid Akbari $^{1}$, Morgan Simpson ${ }^{1}$, Savitri Maharaj ${ }^{1}$, Armando Marino $^{1}$, Deepayan Bhowmik ${ }^{1}$, \\ G. Nagendra Prabhu ${ }^{2}$, Srikanth Rupavatharam ${ }^{3}$, Aviraj Datta $^{3}$, Adam Kleczkowski $^{4}$, J. Alice R. P. Sujeetha ${ }^{5}$ \\ ${ }^{1}$ Faculty of Natural Sciences, University of Stirling, Stirling, UK \\ ${ }^{2}$ Sanatana Dharma College, Alleppey, Kerala, India \\ ${ }^{3}$ International Crops Research Institute for the Semi-Arid Tropics, Hyderabad, India \\ ${ }^{4}$ Mathematics and Statistics, University of Strathclyde, Glasgow, UK \\ ${ }^{5}$ National Institute of Plant Health Management, Hyderabad, India
}

\begin{abstract}
The main objective of this paper to show the potential of multitemporal Sentinel-1 (S-1) and Sentinel-2 (S-2) for detection of water hyacinth in Indian wetlands. Water hyacinth (Pontederia crassipes, also called Eichhornia crassipes) is one of the most destructive invasive weed species in many lakes and river systems worldwide, causing significant adverse economic and ecological impacts. We use the expectation maximization (EM) as a benchmark machine learning algorithm and compare its results with three supervised machine learning classifiers, Support Vector Machine (SVM), Random Forest (RF), and k-Nearest Neighbour (kNN), using both synthetic aperture radar (SAR) and optical data to distinguish between clean and infested waters.
\end{abstract}

Index Terms - Remote sensing, multitemporal image analysis, Sentinel-1, Sentinel-2, water hyacinth, Eichhornia crassipes, wetland, machine learning.

\section{INTRODUCTION}

The synoptic coverage provided by satellites enables water hyacinth (WH) to be distinguished in colour infrared imagery across a temporal scale at multiple different study sites, with further analysis allowing the infestations to be quantified [1]. A previous study monitoring the Gwydir Wetlands, Australia, used images captured from the optical satellite Landsat- 8 to monitor potential WH infestations within the site. The result showed a means of optical surveillance for the aquatic vegetation behaviour at a large spatial scale [2]. A study of aquatic plants using Quickbird multispectral data of Turkey was capable of producing an unsupervised classification accuracy of $83 \%$ when identifying and mapping plant species [3]. Mixed pixels were identified as a main limitation in the procedure.

However, optical data are not always available without cloud cover. This is a strong limitation when we want to achieve a prompt alert system that is able to detect the infesting weed at early occurrences. Synthetic aperture radar
(SAR) can help with this due to the capability to monitor in all-weathers, day- or night-time. The scattering processes of SAR allow mapping of marsh, surface waters and forest to be determined from volume-, double-bounce and surface scattering [4]. Hess et al. [5] used SAR to distinguish between water, herbaceous vegetation and forest within the Amazon Basin.

Multi-modal, multi-sensory approaches to study $\mathrm{WH}$ are generally missing in the literature. The authors of [6] reviewed the latest developments in the use of remote sensing and other technologies to monitor WH infestation. They also proposed a multimodal approach that combines the strengths of the different methods. In this paper, as an extension of our previous study [7], we use an unsupervised machine learning classifier based on the expectation-maximization (EM) algorithm, applied to multitemporal data from S-1 SAR and optical S-2, for detection of WH within Vembanad Lake, Kuttanad, India, and compare its results with three supervised classifiers The results show that the monitoring of WH is possible using only S-1 and S-2 data individually. Fast-flowing currents can rapidly change the position of the free-floating WH and in cases of a time gap between the optical and radar data, the combination of these two data will be challenging. In those cases, we only use one data type (either SAR or optical). We can use S-2 data to fill time gaps in radar observations. This paper presents a quantitative analysis of detection performance using traditional machine learning algorithms with these two data sets.

\section{DATASET AND MATERIAL}

\subsection{Study Area}

Kuttanad, Kerala is a paddy-rich region in south-west India. The Department of Agriculture Development and Farmers' Welfare has reported intensive fertiliser usage by local farmers in the Kuttanad region [8]. This has resulted in an increase in WH found within the major lakes of the region. Due to the 


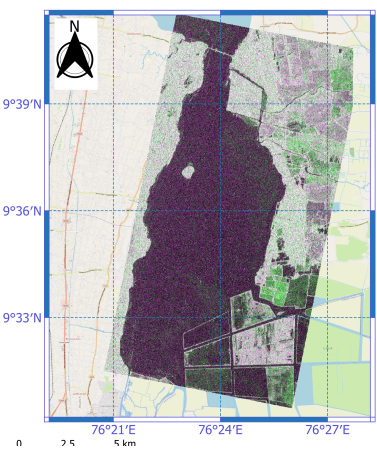

(a)

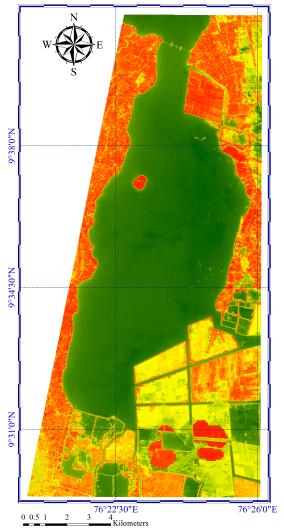

(c)

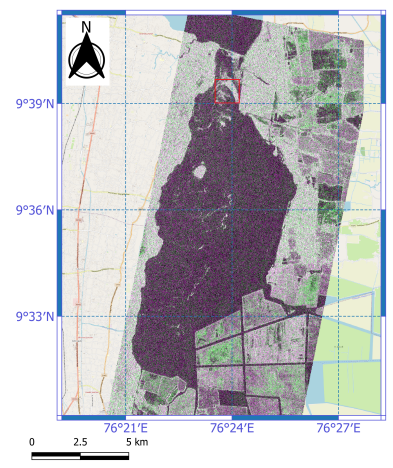

(b)

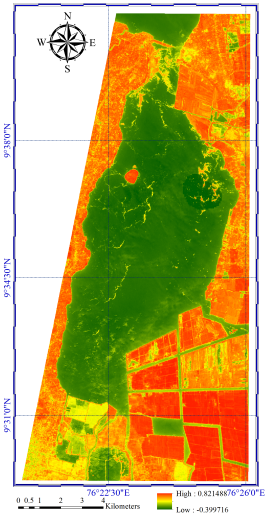

(d)
Fig. 1. False color RGB (VV, VH, VV/VH) composite image (Top) and NDVI (Bottom) of S-1 and S-2 data sets acquired on (Left) Nov-2019 with clean water and (Right) Jan-2020 showing WH on surface of Vembanad Lake.

presence of WH within the region's waterways, impacts have been felt on fisheries, drinking water, irrigation, transport and recreational use of the water bodies. This study focuses on the Vembanad Lake, the largest Ramsar site in Kerala, India.

\subsection{Data Analysis}

Inspecting satellite images for the Vembanad Lake showed that there was clearly something floating on the water surface. Regions of Interest (ROIs) were established to create timeseries of co-polarized (VV) backscatter and cross-polarized (VH) backscatter data. Initial viewing of the time-series data showed that there were clear dates where the VV channel data peaked and troughed. Inspection of these dates showed that, on these dates, the water surface was cleaner, Fig. 1(a), or more infested with WH, Fig. 1(b).

Our hypothesis is that WH infestation alters the scattering by increasing the roughness of the lake surface. This should be distinguishable in the satellite image as spots or patches with high brightness. When smooth, the waters of a lake will scatter most electromagnetic radiation in the specular direction and therefore will appear darker. On contrary, the presence of the non-reflective WH mat on the water surface will

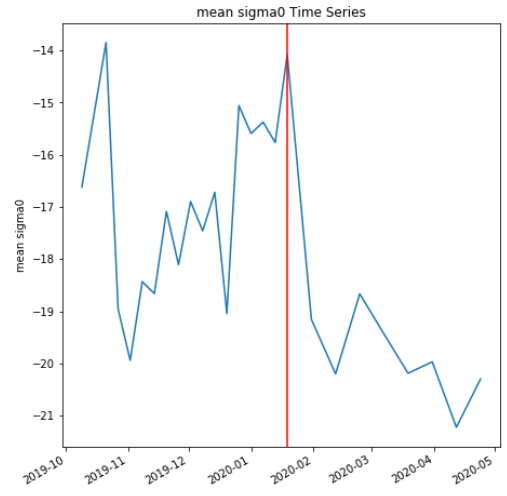

Fig. 2. Time series profile of mean radar backscatter (VV channel) over an infested area.

appear brighter in the image and should be picked up by the radar. As seen in Fig. 2, the radar backscatter of the ROI area increases in infestation times, in particular around January 2020 , indicated by a vertical red line in the time-series profile.

It is observed that the presence of WH will cause the optical reflectance to change and as a result, the Normalized Difference Vegetation Index (NDVI) will increase, as seen in Fig 1(d) in infested time compared to Fig 1(c) in clean time.

\section{METHODOLOGY}

Our workflow for WH detection involves five operations: preprocessing, land masking, feature extraction, unsupervised machine learning classification, and identification of WH.

3.1. Preprocessing: The preprocessing carried out on the input GRD Sentinel-1 data consists of 6 steps: 1) update orbit information, 2) radiometric calibration to obtain sigma0 $\left.\left(\sigma^{0}\right), 3\right)$ thermal noise removal, 4) speckle filtering, 5) range Doppler terrain correction, 6) subsetting, and 7) convert SAR backscatter intensities into decibel-scale $(\mathrm{dB})$. The image pixels were obtained at the final geocoded pixel spacing of $10 \times 10 \mathrm{~m}$ in the Universal Transverse Mercator (UTM) coordinate system.

The Sentinel-2 data were downloaded from the Sentinel2 Global Mosaic (S2GM) service which provides analysisready composites from time-series of Sentinel-2 surface reflectance observations to monitor large area in a timely manner. These data are analysis-ready as typical preprocessing and correction steps have already been applied.

3.2. Land masking: Land masking is important, not only for the obvious reason that only $\mathrm{WH}$ in the open water is of interest, but also because land vegetation can have similar backscatter and spectral signature to $\mathrm{WH}$, producing a high number of false alarms. For this reason, automatic land detection algorithms need to be considered. In this paper, land masking is performed using the SRTM digital elevation model to ensure that WH detection was carried out only on the area 

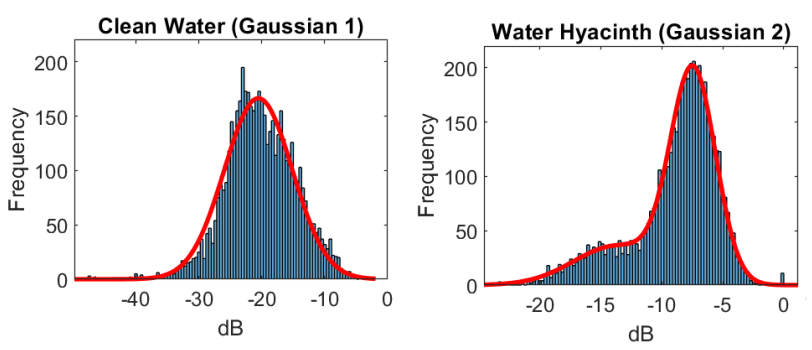

Fig. 3. Parametric Gaussian model fit to the histograms (VV polarization channel). Left and Right are for clean and infested water, respectively.

of interest.

3.3. Feature extraction: A number of optical and SAR features, such as texture features and vegetation indices, which are related to the physical and spectral properties of the scattering medium are extracted. These features are chosen based on the observation that WH exhibits a different backscatter and spectral behaviour compared to the surrounding open water. The features used are as follows:

- Backscatter intensities: $<\left|S_{\mathrm{vv}}\right|^{2}>,<\left|S_{\mathrm{vh}}\right|^{2}>$

- Cross-polarization ratio: $\gamma=\frac{\left\langle\left|S_{\mathrm{vv}}\right|^{2}\right\rangle}{\left\langle\left|S_{\mathrm{vv}}\right|^{2}\right\rangle}$ gives an estimate of depolarization of the SAR signal. The ratio is expected to be higher for WH than clean water.

- $\operatorname{Span}=<\left|S_{\mathrm{vv}}\right|^{2}>+<\left|S_{\mathrm{vv}}\right|^{2}>$

- Gray level co-occurrence matrices (GLCMs) texture features: contrast, dissimilarity and homogeneity, entropy [9].

- S-2 bands were processed to give vegetation indexes, including the normalized difference vegetation index (NDVI), normalized difference Red-Edge (NDRE), normalized difference water index (NDWI), normalized difference aquatic vegetation index (NDAVI), and Soil Adjusted Vegetation Index (SAVI).

The extracted features do to a certain extent have a nonGaussian spreading. The features are therefore transformed, by, e.g., logging the parameters, to make their sample distributions more symmetric and cause their spreading to be closer to a Gaussian distribution. By incorporating this transformation stage, simple segmentation methods, such as a mixture of Gaussians, become applicable.

3.4. Expectation maximization algorithm: We first investigated the potential of statistical parametric approaches by fitting Gaussian probability distribution functions (PDF) on the histograms of different features. The Gaussian models can be expressed as

$$
f(x)=\sum_{i=1}^{n} a_{i} e^{\left[\left(\frac{x-b_{i}}{c_{i}}\right)^{2}\right]},
$$

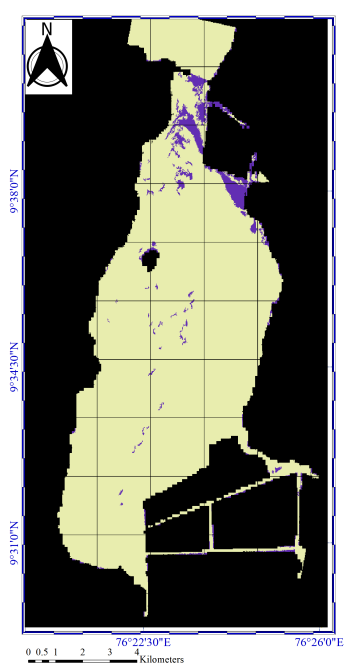

Fig. 4. An example of classification map of infested lake using the proposed algorithm. Purple $=\mathrm{WH}$, yellow $=$ clean, and black $=$ land.

where, $f(x)$ is the distribution for input data $x, n$ is the number of peaks and $a, b$ and $c$ are the amplitude, centroid and width parameters, respectively.

We estimated the Gaussian model parameters by fitting the PDFs on the histograms of the clean and infested areas and the results are shown in Fig. 3. Our study indicates good parametric fit when $n=2$, i.e., mixture of two Gaussian distributions particularly for WH infested area. This is due to the heterogeneous nature (weed and water both) of the region of interest. On contrary, the homogeneous nature of clean water can be well approximated by a single peak distribution. The model parameters, $a, b$ and $c$ shows clear distinction between clean and infested areas.

The unsupervised classification is achieved through a finite mixture model estimated with a pixel-wise EM algorithm [10], where the number of clusters is automatically determined with the goodness-of-fit (GoF) based approach. The initial state and the number of classes were addressed with an automatic strategy by consistently starting as one class and adaptively splitting classes until a statistical criterion is satisfied in the GoF test. In addition, Markov random field (MRF) [11] based contextual smoothing is applied after clustering to improve the smoothness of the final image.

3.5. Identification: The unsupervised machine learning results in an unlabeled map having either one class (clean water) or two classes (clean and infested areas). The labeling step uses the EM output to select the classes corresponding to WH. In the prediction map, we distinguished WH infested areas from water by assuming that they had higher backscatter or higher NDVI values. 
Table 1. Classifier accuracy for different classifiers using the kappa value: (top) S-1 features; (bottom) S-2 features based on validation patches.

\begin{tabular}{|l|l|l|l|l|}
\hline Sensor & EM & SVM & RF & kNN \\
\hline S-1 & 0.90 & 0.88 & 0.89 & 0.86 \\
\hline S-2 & 0.87 & 0.89 & 0.86 & 0.79 \\
\hline
\end{tabular}

\section{RESULTS}

The proposed method first masks out land areas and then uses a feature-based segmentation stage to produce distinct, homogeneous regions corresponding to $\mathrm{WH}$ and clean water. The number of classes in the segmentation algorithm is determined by an automatic strategy based on information contained in the GoF of the data to the estimated model [12]. Figure 4 shows an example of the classification result obtained using the proposed algorithm. The segmentation returns either one or two clusters depending on the existence of infestation in the lake. To evaluate the performance of the proposed method, we identified several training patches from the temporal stack having distinct and homogeneous WH regions. We then classified those patches using three supervised machine learning algorithms, namely, Support Vector Machine (SVM), Random Forest (RF), and k-Nearest Neighbor (kNN) and compared the results with the EM algorithm. For some of the comparison results (comparing classifiers and optical versus radar), the kappa value is used to provide a summary of the classification accuracy. The results are reported in Table 1 . Values of kappa over 0.75 are generally regarded as being indicative of excellent agreement between actual and predicted classes.

\section{DISCUSSION AND CONCLUSIONS}

Using multitemporal S-1 and S-2 data of Vembanad Lake, India, we demonstrated how SAR and optical can be employed to monitor water hyacinth using machine learning techniques. Pixel values have been extracted from ROIs within the lake and show that backscattering intensities and spectral reflectance signatures differ between clean sites and infested sites. Various challenges including accurate coregistration, spectral variation in the time series, missing values for cloudy pixels and time gap between SAR and optical data for floating aquatic vegetation have to be addressed when designing a SAR-optical approach for WH monitoring.

Acknowledgement: This work was funded by the Royal Academy of Engineering under the Frontiers Follow-on Funding scheme (FF9207)

\section{REFERENCES}

[1] J. Everitt, C. Yang, D. Escobar, C. Webster, R. Lonard, and M. Davis, "Using remote sensing and spatial in- formation technologies to detect and map two aquatic macrophytes," Journal of Aquatic Plant Management, vol. 37, pp. 71-80, 1999.

[2] R. Shilpakar, J. Li, L. Ge, P. Dawson, and S. Chapman, "Water hyacinth mapping in gwydir wetlands using remote sensing techniques," in NSW Biennial Weeds Conference, 2017, pp. 87-91.

[3] O. Dogan, Z. Akyurek, and M. Beklioglu, "Identification and mapping of submerged plants in a shallow lake using quickbird satellite data," Journal of environmental management, vol. 90, no. 7, pp. 2138-2143, 2009.

[4] B. Brisco, "Mapping and monitoring surface water and wetlands with synthetic aperture radar," Remote Sensing of Wetlands: Applications and Adv., pp. 119-136, 2015.

[5] L. Hess, J. Melack, A. Affonso, C. Barbosa, M. GastilBuhl, and E. Novo, "Wetlands of the lowland amazon basin: Extent, vegetative cover, and dual-season inundated area as mapped with JERS-1 synthetic aperture radar," Wetlands, vol. 35, no. 4, pp. 745-756, 2015.

[6] A. Datta et al., "Monitoring the spread of water hyacinth (pontederia crassipes): challenges and future developments," Frontiers in Ecology and Evolution, vol. 9, 2021.

[7] M. Simpson et al., "Monitoring Water Hyacinth in Kuttanad, India Using Sentinel-1 SAR Data," in IEEE India Geoscience and Remote Sensing Symposium (InGARSS), 2020, pp. 13-16.

[8] M. Kumari, S. Syamaprasad, and S. Das, "Inland waterway as an alternative and sustainable transport in kuttanad region of kerala, india," in Adv. in Water Resources Engineering and Management, 2020, pp. 245-257.

[9] R. M. Haralick, K. Shanmugam, and I. Dinstein, "Textural features for image classification," IEEE Transactions on Systems, Man, and Cybernetics, vol. SMC-3, no. 6, pp. 610-621, 1973.

[10] A. P. Dempster, N. M. Laird, and D. B. Rubin, "Maximum likelihood from incomplete data via the EM algorithm," J. Roy. Statist.Soc. B (Methodol.), vol. 39, no. 1, pp. 1-38, 1977.

[11] S. Li, Markov Random Field Modeling in Image Analysis. Springer-Verlag, 012001.

[12] V. Akbari and C. Brekke, "Iceberg detection in open water and sea ice using C-band radar polarimetry," in 2017 IEEE International Geoscience and Remote Sensing Symposium (IGARSS), 2017, pp. 2298-2301. 